\title{
Word classes in sign languages
}

\section{Criteria and classifications}

\author{
Waldemar Schwager and Ulrike Zeshan
}

Max Planck Institute for Psycholinguistics, Nijmegen, The Netherlands /

University of Central Lancashire, Preston, UK

The topic of word classes remains curiously under-represented in the sign language literature due to many theoretical and methodological problems in sign linguistics. This article focuses on language-specific classifications of signs into word classes in two different sign languages: German Sign Language and Kata Kolok, the sign language of a village community in Bali.

The article discusses semantic and structural criteria for identifying word classes in the target sign languages. On the basis of a data set of signs, these criteria are systematically tested out as a first step towards an inductive classification of signs. Approaches and analyses relating to the problem of word classes in linguistic typology are used for shedding new light on the issue of word class distinctions in sign languages.

\section{o. Introduction}

This article reports on in-progress research on word class typology across sign languages, which is based on data from two different sign languages: German Sign Language and Kata Kolok, the sign language of a village community in Bali. The goal of this research project is to investigate the parts of speech (PoS) systems in the target sign languages in a way that produces descriptively adequate results for each of the languages, while at the same time developing a cross-linguistically applicable methodology. This is a very challenging task with few precedents in the research literature on sign language linguistics. Not only have there been very few attempts at identifying word classes in individual sign languages, there are also serious theoretical problems that need to be resolved along the way.

Summarising some initial methodological approaches and empirical results, we explore semantic, syntactic and morphological criteria and classifications that enter into a multi-factor analysis of parts of speech systems in sign languages. Although a number of further stages of analysis will be necessary until actual PoS classifications 
can be proposed for the target languages, the theoretical and methodological principles of the approach can already be demonstrated, as well as the kinds of interesting insights, though far from exhaustive, that can be gained along the way. Eventually, further research aims at testing the approach on a wider data set comparing 250 signs from each of the target languages against an exhaustive set of semantic, morphological and syntactic criteria.

This article consists of two main parts: Section 1 summarises a number of theoretical issues that arise when we consider PoS systems in sign languages, and gives a brief overview of previous work on the topic. Section 2 focuses on the comparative study of the two target sign languages, where criteria and classifications are tested against actual sign language data. We discuss the semantic, the syntactic, and the morphological levels of analysis, all of which enter into a comprehensive perspective on PoS classification in sign languages.

\section{Theoretical issues and previous work on PoS systems in sign languages}

\subsection{Sign languages and sign language data}

Sign languages are visual-gestural languages that use the hands and arms as well as non-manual means such as facial expressions, head movements, and body postures to convey linguistic messages. Research over the past decades has demonstrated that sign languages are natural human languages with a complex organisation of their lexicon and grammar. Moreover, the structure of each sign language is independent of the structure of the surrounding spoken language(s). For instance, the sign language used in the German deaf community is lexically, grammatically and typologically very different from spoken German. Most known sign languages are predominantly used by deaf communities in urban settings and exist as minority languages among the majority spoken and written languages used by hearing people. Another, lesser-known situation where sign languages are used concerns village communities with a history of hereditary deafness and a local sign language that is used by deaf and hearing people alike. Both types of sign languages feature in the research that this article reports on.

There is no satisfactory way of recording the dynamic, three-dimensional properties of sign language utterances on paper. In the absence of multi-media publications with integrated video files, authors in sign language linguistics therefore resort to a more or less standardised transcription, which may be enriched by illustrations for added clarity. The transcriptions consist of multi-line, vertically aligned text not unlike interlinear transcriptions in spoken language linguistics. However, the central transcription line consists not of a representation of the signs themselves, but instead employs small-caps glosses as stand-ins for the signs. Glosses are often in English, but may be in another relevant written language. In this article, we generally use German 
glosses for German Sign Language signs and English glosses for Kata Kolok signs. ${ }^{1}$ The transcriptions allow the reader to reconstruct the word order in a signed utterance and the internal morphology of signs, but do not give any indication of what the utterance actually looks like. A number of abbreviations are used in the transcriptions, which are listed in the appendix to the article.

\subsection{The word unit in sign languages}

In order to provide some background to the discussions in Section 2, particularly for the benefit of non-specialists in sign language linguistics, this section characterises the word unit in sign languages and briefly touches on theoretical issues associated with this topic. A number of terms and concepts specific to sign language linguistics are also introduced.

Previous work on sign languages has made considerable progress in identifying and characterising the word unit. Although sign language linguists generally speak of 'signs' rather than 'words', both essentially denote the same kind of entity. Zeshan (2002) argues that for signers, the cultural and psycholinguistic validity of signs is equivalent to that of words in spoken languages, and shows that it is possible to identify grammatical and phonological words, as well as clitics and affixes in sign languages. Sandler (1999) explores the sign unit in terms of a number of constraints that typically apply to monomorphemic signs, thus characterising a canonical sign unit. This includes constraints for the canonical sign to be monosyllabic, that is, having a single movement contour, and to use only one set of selected fingers in its handshape configuration.

A serious theoretical problem at the sub-lexical level is due to the fact that sign languages are much more iconic than spoken languages, with many signs "looking like" what they mean to some degree. In fact, sign languages can be characterised as languages with a very high degree of phonosymbolism (Zeshan 2002). ${ }^{2}$ When a sign meaning 'tree' or 'write' (see Figures 1 and 2) visually represents parts of the concept

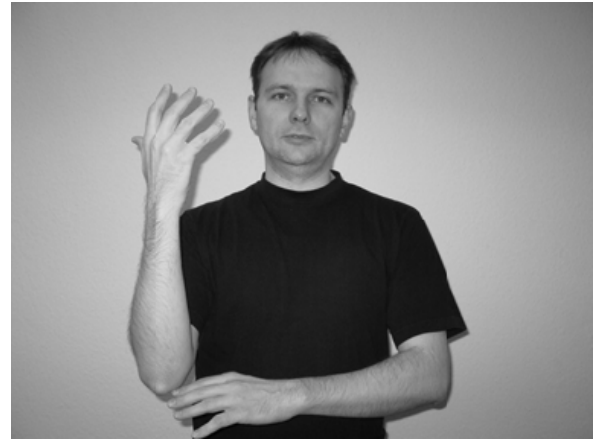

Figure 1. BAUм ('tree') in German Sign Language.

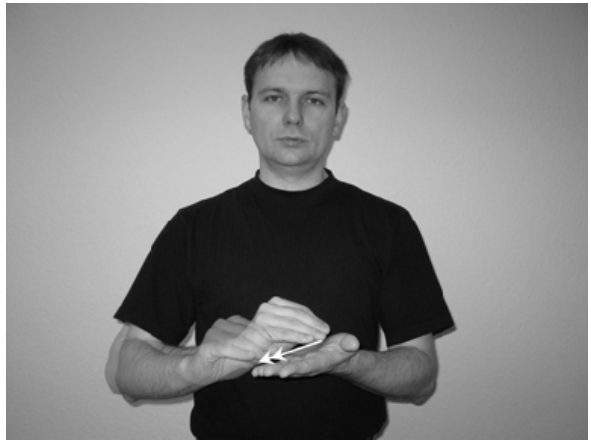

Figure 2. SCHREIB ('write') in German Sign Language. 
on the hands - for instance, the tree trunk by the lower arm and the tree branches by the fingers, or the paper to write on by the palm of one hand over which the other hand moves as if to write on it - this can cause confusion because these parts of the signs would be minimal meaningful units, but would otherwise be regarded as phonemes rather than morphemes.

Usually, a sign is considered to consist of several formational units, its phonemes or phonological parameters: handshape, movement, place of articulation, hand orientation, and sometimes a non-manual component. The sign in Figure 1 therefore has a handshape phoneme (all fingers extended), a movement phoneme (wrist twisting), a place of articulation phoneme (space in front of the signer), and so on. However, it is also clear at the same time that the fingers of the hand represent the branches of the tree, and signers can exploit this iconic potential. For instance, the branches (fingers) could be made to sway in the wind, or the other hand, representing a bird, could make contact with the first hand to perch on a branch (e.g. the little finger) of the tree. The handshape is then clearly meaningful in such signs while, at the same time, continuing to function as a building block of the sign at the phonological level. This overlap between formational and meaningful functions of sub-lexical parts of signs causes theoretical problems in distinguishing phonemes from morphemes in sign languages, which has led some authors to coin new terms such as "phonomorphemes" or "ion-morphs" (Fernald and Napoli 2000). On the other hand, Zwitserlood (2003) argues that signs such as the ones discussed here should indeed be regarded as morphologically complex.

At the current stage of research, there is no widely accepted overt definition of the morpheme unit in sign languages, although it is clear that the traditional notion of morphemes as being "minimal meaningful units" is problematic to apply to sign languages. In the absence of such a definition, it is methodologically preferable to start by investigating morphological processes, which are relatively straightforward to identify. This is the approach used in this article, particularly in Section 2.5.

While we normally have little difficulty to identify the words/signs in signed utterances, there are cases where this is problematic. In particular, this concerns certain types of spatial-iconic signs that are usually called "classifiers" in the sign language linguistics literature (cf. Schembri 2003). ${ }^{3}$ The analytical problem is most conspicuous in a sub-type where particular handshapes represent classes of similar referents. For instance, in German Sign Language an upright index finger PERSON-CL is used for human referents, whereas a horizontal flat hand can represent vehicles (Figures 3 and 4).

Since both hands can be used simultaneously for signing, it is also possible to combine these two signs into one, with the entity in the class of vehicles (e.g. a car) represented on the right hand and an entity in the class of human referents (e.g. a man) represented on the left hand. The movement and location of the classifier hands iconically map onto the movement and location of their referent entities, and these constructions can be used very productively. For example, the right hand could well be 


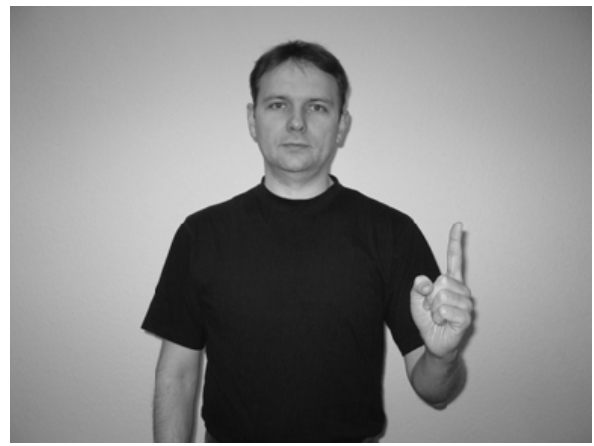

Figure 3. PERSON-CL in German Sign Language.

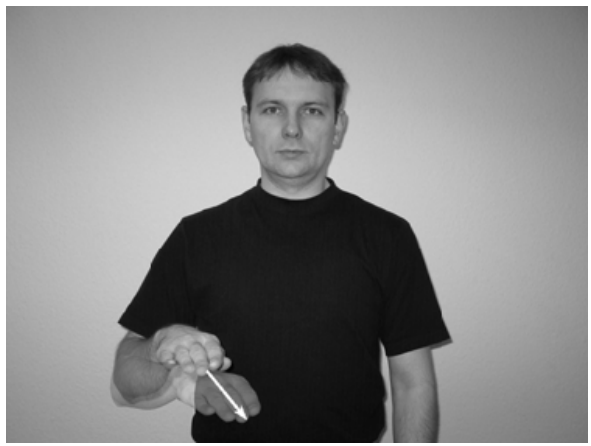

Figure 4. VEHICLE-CL in German Sign Language.

"approaching" from one side and then "crash into" the other hand, as in the man being run over by the car. It is very difficult to decide how many signs/words such a sequence should be segmented into. Is each hand a word, and both occur simultaneously? Would the "approaching" part of the hand's movement be a separate word from the "collision" part of the movement? Is the whole proposition contained in a single sign/word, or is it just difficult to identify the word boundaries in such an utterance? To then give a convincing account of the internal morphological structure of such formations is even more difficult, and they have long resisted a convincing morphological analysis in sign language research (cf. Emmorey 2003 for an overview). For the purpose of this article, classifier signs are not considered in the analysis, but it should be kept in mind that parts of the lexicon in sign languages are theoretically challenging to deal with and eventually will have to be included in a comprehensive word class analysis.

We also find a substantial amount of systematic ambiguity or vagueness in many sign languages. For instance, in Indo-Pakistani Sign Language (IPSL) many signs tend to have rather general meanings that are narrowed down by the context of the utterance, and since many grammatical categories can remain unmarked, a sequence of signs can be difficult to categorise structurally, although its meaning is entirely clear semantically. For example, an IPSL utterance such as in example (1) can have three related, but structurally different interpretations, as given in (2). Note that in IPSL, a personal pronoun can function as a possessive pronoun and nominal plurals need not be marked in any way. Personal pronouns in sign languages are indexical signs (therefore glossed as INDEX) that are oriented towards a location in the signing space, most commonly realised as pointing with the index finger.

IPSL

(1) INDEX 1 WORK A-LOT

(2) a. 'I work a lot.'

b. 'My work is a lot.'

c. 'I have a lot of things to do (works).' 
Although the utterance in (1) is systematically ambiguous as far as the relationship of the individual signs to each other is concerned, this does not mean that the signs in such utterances are thrown together in an ad hoc fashion and lack structural restrictions. In fact, changing the word order as in example (3) results in an ungrammatical utterance:

IPSL

(3) * ${ }^{\text {INDEX }} 1$ A-LOT WORK

The problem with respect to a PoS analysis is that even where we can observe syntactic restrictions in sign languages, it is often far from clear how exactly such regularities relate to word classes, or are criterial for determining the PoS system. Thus the above data from IPSL do not provide clear clues as to whether the sign wORK has a nominal or a verbal character, and similar problems are encountered in many other sign languages as well.

Where morphological marking occurs in sign languages, it is similarly often difficult to understand how it may relate to or be criterial for establishing word classes. Morphological processes are often inapplicable for phonological reasons, that is, because of the physical form of the sign. For instance, if a morphological process requires a sign to be repeated at several locations in space rather than being signed only once in its neutral location, the process by definition cannot apply to signs that must be made on a particular part of the body, such as signs touching the head or the chest area. The types of morphology that we commonly find in sign languages are uncommon in most spoken languages (cf. Section 2.5), and their restrictions can operate in ways that do not obviously relate to criteria for word classes.

\subsection{Previous approaches to PoS classification in sign languages}

In the description of undocumented spoken languages, determining the word classes and their properties is typically one of the first tasks. By contrast, sign language linguistics has been quite shockingly neglectful of this fundamental issue. Generally, signs are either labelled as "adjectives", "nouns" etc. on the basis of a translation of the sign's meaning into a relevant spoken language - DEAF in British Sign Language must be an adjective because deaf in English is an adjective - or the PoS system of American Sign Language (ASL), the most extensively documented sign language, is uncritically applied to a lesser-described sign language. Both approaches are, of course, neither methodologically nor theoretically viable.

Principled attempts at PoS classification in sign languages are few and far between. An early influential study on ASL discusses the distinction between related pairs of nouns and verbs (Supalla and Newport 1978). In ASL, there are subtle differences in movement patterns between sign pairs such as AIRPLANE and FLY-BY-AIRPLANE, SCISsors and CUT-WITH-SCISSORS, DOOR and OPEN-DOOR, and the like. The handshape, 
hand orientation and place of articulation are the same in both signs of each pair, but the movement patterns differ, with nouns (AIRPLANE, SCISSORS, DOOR) being characterised by smaller and repeated movements. This distinction holds for instruments and related actions only, such as in SCISSORS and CUT-WITH-SCISSORS, and does not apply to all nouns and verbs in ASL. Johnston (2001) investigates whether the same process applies in Australian Sign Language as well. Padden's (1988) seminal study on ASL discusses a number of diagnostic tests and argues for a PoS system including nouns, adjectives, and three sub-classes of verbs. In simplified terms, the main criteria are the following (note the starred ungrammatical examples in (4)-(6) below): Nouns can be modified by quantifiers, as is exemplified by TREE in (4a), which is quanitified by the sign FOUR. By contrast, a verb such as RUN cannot be combined with a quantifier. Adjectives are defined by being able to inflect for intensive aspect. The form of a sign such as RED can be formationally modified and then means 'very red' (5a). By contrast, TREE cannot be modified in this way (5b). Finally, verbs cannot be pre-modifiers of other signs, so whereas the adjective BIG is modifying sHOE in (6a), the verb RUN is ungrammatical in the same modifier slot (6b).

\section{ASL}

(4) a. FOUR TREE

b. ${ }^{*}$ FOUR RUN

(5) a. RED\#INTS

b. * TREE\#INTS

(6) a. BIG SHOE

b. ${ }^{\star}$ RUN SHOE

Without going into the details of the analysis here, it is significant to point out that the class of verbs is defined negatively only, through inability to pre-modify other signs. No convincing set of criteria is discussed that would positively identify all sub-classes of verbs, and they in fact have quite different morphological properties (for further details on verb sub-classes, see Sections 2.4.1 and 2.5). Moreover, it is doubtful whether the criteria really apply across the board to all signs that are intended to be members of each category, since they are not systematically tested against a wide variety of lexemes in ASL.

Although the above criteria are clearly language-specific to ASL, they have often been transferred to other sign languages. By contrast, Zeshan (2000) is an attempt at identifying both open and closed word classes in IPSL in a different way, since diagnostic tests employed by Padden (1988) generally do not work for IPSL. The proposed PoS system for IPSL is quite different from Padden's, classifying signs from open word classes in terms of their spatial behaviour. Thus signs are either unable to take any spatial modification, for instance because they must always be made on a particular part of the body, or the place of articulation of a sign can be moved in space, for instance 
by placing the hand on one side of the signer rather than in front, or the sign moves between two grammatically relevant locations. The third class of signs has verbal properties (cf. the discussion of directional/agreement signs in Section 2.5). The PoS classification for IPSL also includes closed word classes such as signs with classifier handshapes and indexical signs. Finally, Erlenkamp (2000) proposes a PoS classification for German Sign Language. We will deal with her proposal in more detail below, since this article also considers German Sign Language as one of its target languages.

In summary, the main problem in all previous PoS analyses of sign languages is that there is no principled way of identifying criteria for PoS differentiation. Criteria seem ad hoc and generally lack theoretical justification, a problem which is aggravated by the fact that semantic, morphological and syntactic criteria may themselves lead to conflicting results (cf. Sasse 1993). While a detailed study of PoS systems in sign languages will need to go beyond traditional categorisations, the alternatives that would lead to descriptively adequate, methodologically sound and theoretically interesting analyses have not been worked out yet.

\section{Towards unified criteria for PoS differentiation in sign languages}

\subsection{Sign language data}

In order to systematically approach some of the theoretically and methodologically challenging issues addressed in the first part of this article, we mainly use data from two unrelated sign languages: German Sign Language (Deutsche Gebärdensprache, henceforth abbreviated DGS), the language of the German deaf community, and Kata Kolok (henceforth abbreviated KK), a sign language used in a village community in northern Bali, Indonesia. These sign languages are not only geographically and genetically unrelated to each other and have never been in contact with one another, they are also typologically dissimilar and exist in quite different socio-linguistic settings. Such a cross-linguistic perspective is important and integral to the approach if there is to be any chance of obtaining insights that are applicable to more than one particular sign language. In addition to these theoretical considerations, the choice of our data has also been motivated by the availability of suitable sign language corpora.

DGS is used as a first language by more than 80,000 deaf sign language users in Germany, as well as about 300,000 people who are hard of hearing. The German deaf community is a linguistic and cultural minority which is in a language contact situation with the surrounding dominant language, spoken/written German. Prior to the language's official recognition in 2002, DGS faced a long period of linguistic oppression, but is now reasonably well documented, including the availability of educational materials for teaching the language. Heßmann (2001) recorded spontaneous signed texts in DGS and transcribed one hour of these data, though in a semantically-based way and without any reference to the part-of-speech assignment of individual signs 
in this corpus. By contrast, Erlenkamp (2000) is an explicit attempt at characterising the word class system of DGS in a typological framework. However, this contribution remains preliminary and has not been widely absorbed in the research community. It has also been criticised for methodological and theoretical shortcomings by Keller, Pfau and Steinbach (2002).

Unlike DGS in its largely urban context, Kata Kolok (literally "deaf language") is the sign language of a small village community in northern Bali. The village has a high incidence of hereditary deafness, with currently 50 deaf people in a total population of about 2,500. ${ }^{4}$ According to oral history, village records and local myths, KK has existed in the village for 800 years (Marsaja 2008). KK is used by both deaf and hearing people in the village, with the deaf being monolingual users of $\mathrm{KK}$ and most hearing people growing up bilingually (or rather, multilingually) with competence in both the sign language and a number of spoken languages used in the village. The KK data corpus was gathered during two field trips as part of the "Sign Language Typology" research project, and the data are archived at the Max Planck Institute for Psycholinguistics (MPI) in the Netherlands. Out of this extensive corpus of spontaneously produced sign language data, four hours of transcribed data are available for the purpose of our comparative parts of speech analysis. Unlike for DGS, there is no research into word classes in KK yet, and its linguistic documentation has only just begun. Previous publications on KK (e.g. Branson, Miller and Marsaja 1996) focus on its socio-cultural setting and do not include information about its linguistic structures.

In Section 2.5, we will use data from a third sign language, Russian Sign Language (RSL), for further comparison. RSL data are also part of the MPI sign language corpora, and the language has been analysed at the morphological level in Schwager (2004). However, RSL data play a limited role within the scope of this work because DGS and RSL are typologically similar to each other, both being in contrast with KK. Parts of speech analyses of the three sign languages function as a pilot project, and it is expected that the resulting framework will be applied to other sign languages in the future.

\subsection{Theoretical background on semantic and structural criteria}

All lexical units of a language have a number of features that characterise them. Words with the same, similar, or equivalent characteristic features can be assigned to the same word class. Therefore, in order to determine the word classes in any given sign language, we also need to study the individual features that characterise its words/ signs. In principle, there are unlimited possibilities of assigning lexical units to classes. Among these possibilities, some are sensible choices and many others are not. How many classes are to be posited depends on the constellation of criteria that are potentially available for the purpose of PoS classification in a particular language, and ultimately depends on the explanatory goal of the analysis itself. 
In this article, we adopt a theoretical framework according to which word class assignment in any given sign language has to be done language-specifically, yet should be based on cross-linguistically applicable criteria. This is an important theoretical decision, which is particularly suitable for comparing typologically diverse languages (cf. Croft 2001). Rather than just imposing ready-made traditional labels such as "noun", "verb", etc., as has been common practice in much of the earlier sign language research literature, it is important to find a principled standard of comparison and apply this consistently across the target languages. Logically, this analysis is done in two stages: 1 . establishing the same, similar, or equivalent distinguishing criteria that are potentially relevant to PoS systems in DGS and KK, and 2. working towards a comparative analysis of PoS distinctions in DGS and KK on the basis of these criteria.

In the process of positing features that are criterial for word class assignment in sign languages, we choose features that are relevant not only for sign languages, but also for spoken languages. The parts of speech analyses generally draw on theoretical work by Sasse (1993) and Anward (2001). Sasse (1993) states that (lexical) categories are language-specific bundles of features which have both formal and conceptual aspects. Therefore, semantic, morphological and syntactic levels of word units are distinguished and enter into a parts of speech analysis in sign languages.

For the semantic level, new semantic feature bundles have been compiled and refined for each concept class (see Section 2.3.2), largely following approaches in Löbner (2002), Sasse (1993) and Anward (2000, 2001). The syntactic level relies on Hengeveld (1992) and Anward (2000), and on the morphological level, criteria were created on the basis of the existing sign linguistics literature, in particular Sandler and Lillo-Martin (2006) (see Section 2.5).

When discussing the assignment of signs to classes, it is important to always be precise about which of these levels is relevant at the time. All too often the morphological level is confused with the semantic or the syntactic level. In order to avoid confusing definitions, classes should only be defined using features that belong to one and the same level. Moreover, it will be preferable for all distinguishing features to be binary. Such binary distinguishing features should not only be posited as such, but should also be structured hierarchically within a particular feature bundle of a class, as is exemplified in Section 2.3.2 below. For PoS differentiation in signed/spoken languages, the following priority of levels is assumed: 1 . Semantic criteria should be determined language-independently and are used as a first step towards PoS differentiation. 2. Syntactic, morphological, and discourse-pragmatic criteria should be determined language-specifically.

It has long been recognised that semantic criteria are problematic as a starting point for assigning words to parts of speech. Thus, Evans (2000) argues that synonyms may be mapped onto different word classes in different languages, so that two words from different languages which essentially express the same meaning may belong to distinct word classes in each language. In fact, such cases are also found in sign 
languages, as we illustrate with examples in Section 2.4.2. However, this line of argumentation only focuses on the lexical level, not on the level of minimal semantic features. Semantic mapping of individual lexical units is of course language-specific, and also sometimes culturally determined, but the minimal semantic features such as [human], [concrete], [individuated], etc., are cognitive-linguistically and, so to speak, pre-categorically based, and can therefore be taken to be language-independent. Thus, they constitute a good point of departure for defining the membership of parts of speech across languages.

Since for sign languages, in particular previously undocumented ones such as $\mathrm{KK}$, the issue of PoS distinctions is severely understudied, it is preferable to begin our pilot study by relying on the widest possible range of criteria, certainly including structural criteria from both syntax and morphology. The ad hoc application of both syntactic and morphological criteria for PoS differentiation in sign languages has proved equally problematic or has produced ambiguous results in one way or another in previous studies. For instance, one of the findings in both Erlenkamp (2000) and Zeshan (2000) is that most signs in DGS and in IPSL can appear either in an argument or in a predicate slot, without any formal marking. In many spoken languages, nouns and adjectives can function as predicates without any verb or copula needed in the sentence, and this is also the case in sign languages. By and large, it has not been easy to identify workable syntactic tests for sign languages, given that they often have relatively free word order and some of their sentence structures are unfamiliar from a spoken language background, including spatial syntax and simultaneous constructions. We still do not know enough about the syntactic behaviour of sign languages to rely on syntactic criteria exclusively.

Distinguishing word classes on the basis of morphological criteria is possible only for languages that have appropriate processes of inflection and/or agglutination. For isolating languages such as Chinese, PoS-classes cannot be posited on the basis of morphological criteria. Sign languages also have a large number of such non-inflecting lexemes, as noted in Erlenkamp (2000) for DGS. Moreover, morphological criteria are always language-specific and may be impossible to apply across sign languages in their entirety if a certain morphological process occurs in one sign language but is absent in another sign language (see examples from DGS and KK in Section 2.5). Morphological processes by themselves do not constitute reliable criteria for word class assignment in sign languages. ${ }^{5}$

In the remainder of this article, we posit syntactic and morphological criteria in combination with semantic criteria, and then test the viability of this approach against examples from the sign languages in our corpus, mainly focusing on Kata Kolok and German Sign Language. 


\subsection{Semantic criteria for PoS differentiation}

\subsubsection{A semantic metalanguage}

Given that semantic criteria are taken as a starting point in our approach, the next question to deal with is which semantic criteria should be identified to establish the intended semantic metalanguage. The concepts of our cognitive world fall into various classes, yet such semantic classification is not random across languages because of a degree of language-independent, cognitive-linguistic determination. Similar concepts can be grouped together in a class, which is called a concept class. In Sasse's terms (1993: 202): "Eine Konzeptklasse ist ein Bündel verwandter Konzepte, das durch ein übergeordnetes Merkmal (gewöhnlich ein „Hyperonym“) definiert werden kann.” 6 Such classes, by virtue of their semantic features, often figure in lexical-grammatical restrictions related to parts of speech in a given language. At the semantic level, concept classes such as concrete entities, abstract entities, properties, states, actions, etc, can be posited. Expanding on Stassen (1997), Anward (2000, 2001) defines a total of eight semantic classes: person/thing, ${ }^{7}$ event, property, place, time, relation, quantity and situation. However, in order to approach a characterisation of lexical meanings that can be used cross-linguistically as a starting point for our analysis, we need to take another step and also consider bundles of minimal semantic features that characterise a semantic class, as well as inherent properties of the semantic features (which may themselves be either categorical or gradual).

According to Löbner (2002), distinguishing features of concept classes should always be binary. In this regard, it is important to note that not all features are applicable to all concept classes. For example, a binary feature [ \pm dynamic] that defines an aspectual differentiation between 'dynamic' and 'static', is fully compatible with the semantic class of events, but is not really applicable to the entity class. Therefore, in tabulating the minimal semantic features of concept classes, the feature [dynamic] can be positive or negative within the concept class of events, but is zero, that is, inapplicable, within the concept class of entities (cf. Table 1 in Section 2.3.2).

Moreover, semantic classes characterised by bundles of distinguishing features should not show fuzzy boundaries. Following Löbner (2002), we assume that semantic classes or categories have an internal structure, with better and less good (in other words: more or less 'prototypical') members of a category, and that sign/word meaning can in addition be flexible/vague. However, the boundaries of a given semantic category cannot be fuzzy and membership of lexical items in semantic categories is not gradient, but binary. In other words, semantic classes/categories have variable, but not fuzzy boundaries. Finally, defining a structured hierarchy for the minimal semantic features is crucial for well-formed semantic categorisation. For instance, it is logically impossible for a feature [human] to be higher than a feature [animate]. Section 2.3.2 provides examples of such feature hierarchies. 
Within the framework of this semantic metalanguage, the fact that there are better and less good members of a concept class is not unexpected, and may be explained with reference to diachronic reasons, such as grammaticalisation and lexicalisation. Such units may partially adopt new features of a concept class but still preserve some previous features of another class. Thus, semantic change may, for certain lexical items, cause semantic "interference" with a purely synchronic classification of a language, but does not jeopardise the approach in general.

As is detailed in the following section, semantic features and concept classes have been posited with a view towards being cross-linguistically valid, so that a typological comparison of languages at the semantic level in terms of same, similar or equivalent units becomes possible. Although the aim of establishing the hierarchical semantic features as such is to obtain a valid cross-linguistic basis, this does not mean that all features have to be present in all languages. Rather, certain features can be lexicogrammatically relevant in one language, but irrelevant, and therefore absent, in another language. For instance, the distinction between what is traditionally known as "mass nouns" and "count nouns" is grammatically relevant in English and in American Sign Language (Padden 1988), but not in Indo-Pakistani Sign Language (Zeshan 2000) and in many spoken languages. Similarly, Wilbur (in press) claims that the semantic feature of telicity is reflected in formational aspects of signs in American Sign Language, but this is unlikely to hold in all sign languages. ${ }^{8}$

In principle, a language-specific, synchronically adequate system of semantic features can be deduced from the general, language-independent semantic metalanguage, although for the purpose of this article this has not actually been done yet. Rather, we explicate selected parts of the semantic feature system regarding the concept classes of entity, event and property. The event class is itself subdivided into (sub)classes of action, process and state. There are further concept classes to be identified which do not enter into the discussion here, such as: time class (e.g. 'tomorrow'), place class (e.g. 'here'), quantity class (e.g. 'three,' 'much'), and some synsemantic classes (such as deictics, classifiers, etc.).

\subsubsection{Semantic feature systems for entity, event, and property classes}

To exemplify a concrete instantiation of the theoretical considerations explained in the previous section, this section proposes partial semantic feature hierarchies for the event and the entity classes, as well as the more problematic property class. We then take a look at a number of semantically equivalent signs from DGS and KK to test how the semantic metalanguage functions in the cross-linguistic comparison of signs.

Figure 5 shows a feature hierarchy for the entity concept class. The feature system partly follows the traditional practice of ontological classification known since the time of Aristotle and Dionysius Thrax (cf. Lehmann and Moravcsik 2000), but also includes some new, refined features in the hierarchy. A systematic matrix of hierarchically structured semantic features, as given in Figure 5 for the entity class, and also in 
Figure 6 for the event class, is not commonly found in the literature. While many of the labels are well-known, they have been re-arranged into comprehensive matrices of semantic features. Lehmann and Moravcsik (2000) group nouns into subclasses with a view towards their morphosyntactic behaviour, and their subclasses include categories such as mass nouns vs. count nouns, collective vs. individual nouns, and the like. In Figure 5, however, these and other additional categorisations are reinterpreted and cast in terms of minimal semantic features.

In Figure 5, the feature [ \pm proper] first divides this class into common names, designating a concept, and proper names, designating an individual (cf. Lehmann and Moravcsik 2000). Proper names are further subdivided into subclasses by features [ \pm animate] and [ \pm human], yielding classes for person names, individual names for animals, and proper names for things. Under the category of proper names, person names include items such as John, Mary, Gandhi, etc. By contrast, the category of human names (persons) is classified under common names and consists of items such as student, president, baby, dwarf, foreigner, etc.

Using a further feature [ \pm local], proper names for things may be subdivided into geographic names (e.g. Siberia, Berlin) and individual names for objects (e.g. Eiffel Tower, Crown Jewels). As detailed below, place names are particularly interesting with respect to the cross-linguistic comparison of DGS and KK. Whereas the 'proper' class always has the values [+concrete], [0 homogeneous], [+individuated], and [-count], these features are differentiated in the 'common' class. For example, the 'mass' class consists of a sortal subclass (e.g. wine, which has a plural wines) and a material subclass (e.g. air, rice), which are differentiated by a feature [ \pm count $]$.

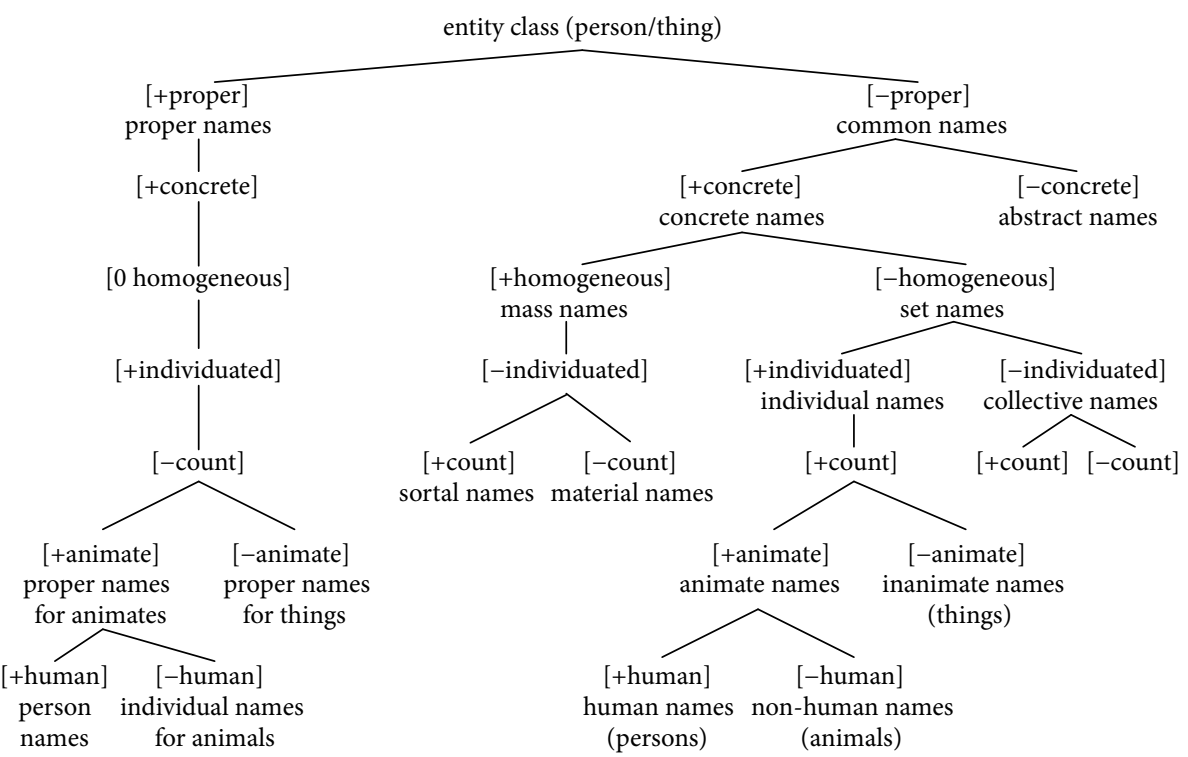

Figure 5. Hierarchy of semantic features for entity class. 


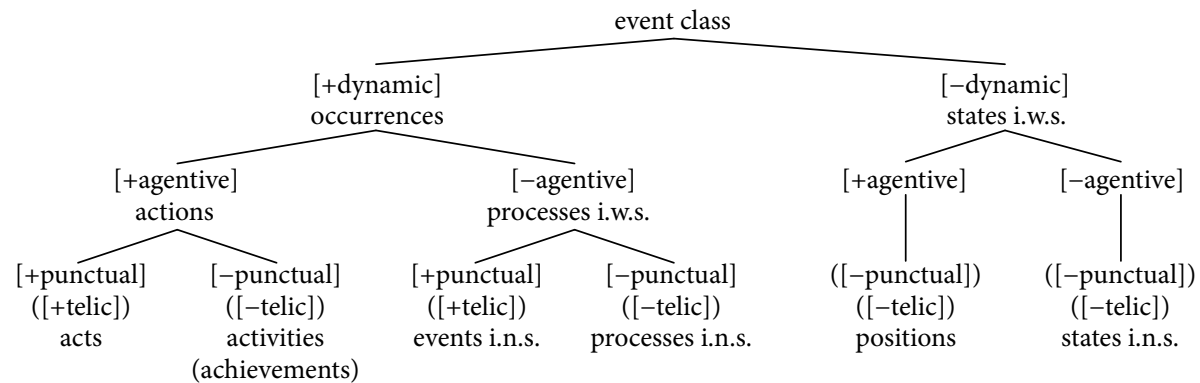

Figure 6. Hierarchy of semantic features for event class.

The event class is subdivided into the (sub)classes of state, action, and process by the features $[ \pm$ dynamic] and [ \pm agentive] (cf. Figure 6 ). Other features used in the event domain focus on telicity and on Aktionsart, drawing on previous theoretical frameworks, in particular Vendler (1967) and Van Valin (2001). Using the additional features of $[ \pm$ telic] and $[ \pm$ punctual], we can characterise signs from our data as belonging to one of the lowest-level sub-classes. The following examples are from DGS: ${ }^{9}$

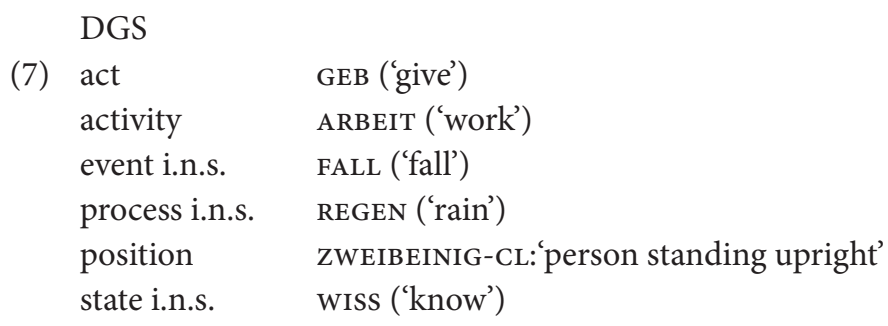

Note that in the previous literature, there is some conceptual overlap between the aspectual feature pair 'static' vs. 'dynamic' on the one hand and the temporal feature pair 'time-stable'/'persistent' vs. 'temporary'/'transitory' on the other hand (e.g. Lehmann and Moravcsik 2000). However, both of these feature pairs are distinct from each other in that the former basically refers to the presence or absence of a change, such as movement or development, in an event concept (cf. also Comrie 1976; Bybee 2000), whereas the latter can refer to the temporal characteristics of any concept class, be it an entity, property, event or any other concept class (cf. Stassen 1997). Thus, the scale of dynamicity may partially overlap with the scale of time-stability inasmuch as the latter applies to event concepts only.

It is more difficult to posit minimal semantic features in the domain of property concepts. The property class can partially be defined on the basis of Dixon's (1982) pioneering work on adjectives inasmuch as there are more or less prototypical semantic types designating properties. Dixon (1982) identifies the following, listed here with DGS signs instantiating each of the types: 
DGS

(8) dimension

value

GROSS ('large')
GUT ('good')
ROT ('red')
NEU ('new')
KALT ('cold')
FRECH ('cheeky')
SCHNELL ('fast')

However, to abstractly define 'property' in terms of basic semantic features is very problematic. For the purpose of this article, a non-aspectual feature [ \pm qualitative] is used to preliminarily distinguish the property class from other classes. This feature applies positively to a property concept but always negatively to any concept from another concept class. In contrast with the conceptual class of states, the property class is, for logical reasons, not easily compatible with aspectual features such as [dynamic] or [punctual], which helps to distinguish the two classes. A further feature [ \pm gradable] subdivides the property class into gradable/polar and non-gradable/absolute property subclasses.

Compiling the abovementioned features of entity, event, and property classes into a single feature matrix, the semantic distribution of features across these three main classes can be read off Table 1. As mentioned before, a 'zero' value means that a feature is not logically compatible with a concept class. This usually means that the feature can be neglected in further analysis because we do not expect it to have any lexical or grammatical relevance for the concept (class) in question.

Table 1. Distribution of semantic features across entity, event, and property classes.

\begin{tabular}{llllll}
\hline $\begin{array}{lllll}\text { binary semantic } \\
\text { features }\end{array}$ & entity & event & property \\
\cline { 3 - 5 } [proper] & \pm & 0 & 0 & 0 & 0 \\
[concrete] & \pm & $0 /-$ & $0 /-$ & $0 /-$ & $0 /-$ \\
[homogeneous] & $\pm / 0$ & 0 & 0 & 0 & 0 \\
[individuated] & \pm & 0 & 0 & 0 & 0 \\
[countable] & \pm & 0 & 0 & 0 & 0 \\
[animate] & \pm & 0 & 0 & 0 & 0 \\
[human] & \pm & 0 & 0 & 0 & 0 \\
\hline [dynamic] & 0 & + & + & - & $0 /-$ \\
[agentive] & 0 & + & - & \pm & $0 /-$ \\
[punctual] & 0 & \pm & \pm & - & 0 \\
\hline [qualitative] & - & - & - & - & + \\
[gradable] & - & \pm & \pm & \pm & \pm \\
\hline
\end{tabular}




\subsubsection{Application of semantic criteria to DGS and KK}

The minimal features in Table 1 are complementarily distributed over the three main semantic classes, and this semantic distribution of the main concept classes tests successfully on data from both DGS and KK. Thus, the featural mapping provides a good point of departure for defining word classes across signed (and potentially also spoken) languages.

Methodologically, one of the main reasons for proceeding via a semantic metalanguage on the way towards our word class typology across sign languages is to facilitate the creation of the basic 250 -word data sets mentioned in the introduction. In deriving the lexical items that will go into each of the data sets, it is important a) to cover all the main conceptual categories, including those not discussed in detail in this article, and b) to choose, as far as possible, signs with similar or equivalent semantic content from all target sign languages. The semantic classifications discussed in this section make it possible to establish data sets that fulfill these two criteria in a principled way.

Though testing the semantic features on signs from DGS and KK has not been done comprehensively for the purpose of this article, a preliminary survey reveals many parallels, but also some interesting differences. Without prejudice to culturallybased semantic differences of lexical meanings in both sign languages, many entity, event and property concepts do overlap in the two languages, as in these examples:

(9)

\begin{tabular}{lll}
\multirow{2}{*}{ entity } & DGS & KK \\
& GEHÖRLOS & DEAF (see Figures 7 and 8) \\
FRAU & FEMALE \\
HUND & DOG \\
REIS & RICE
\end{tabular}

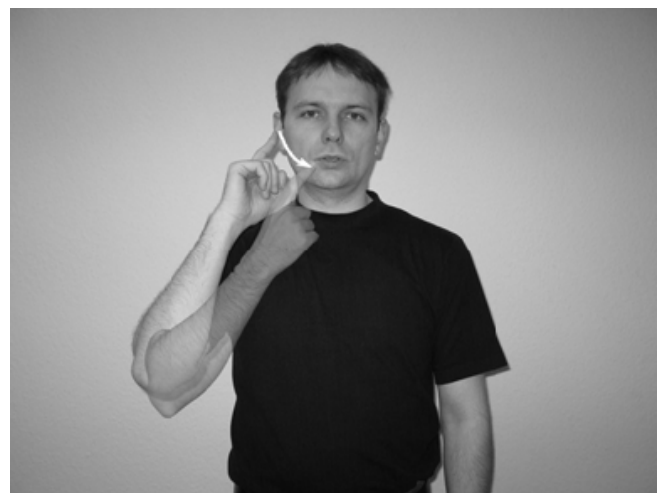

Figure 7. GEHÖRLOS ('deaf') in German Sign Language.

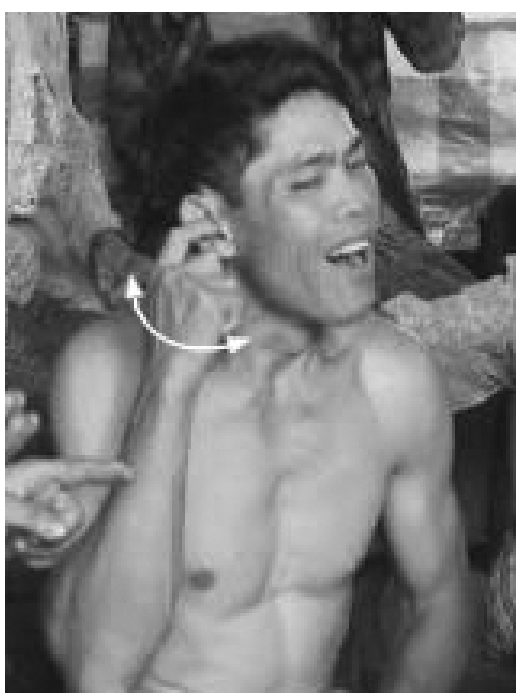

Figure 8. DEAF in Kata Kolok. 


$\begin{array}{lll}\text { event } & \text { ARBEIT } & \text { WORK } \\ & \text { ESS } & \text { EAT } \\ & \text { STERB } & \text { DIE } \\ \text { property } & \text { GEBÄRD } & \text { SIGN-TALK } \\ & \text { SCHLECHT } & \text { BAD } \\ & \text { GROSS } & \text { BIG } \\ & \text { SCHWARZ } & \text { BLACK } \\ & \text { SCHÖN } & \text { BEAUTIFUL }\end{array}$

So far, it seems that differences occur both at the level of individual lexemes and at the level of sub-classes. For instance, the sign DEAF in KK always refers to a 'deaf person' and is an entity concept only. In DGS, the equivalent sign GEHÖRLOS ('deaf') refers to either a person or a property of a person and is therefore used both with an entity and as a property meaning. Consequently, the DGS corpus (Heßmann 2001) includes utterances such as GEHÖRLOS FRAU ('deaf woman'), with GEHÖRLOS being used to refer to a property of an individual. Despite the very frequent occurrence of the sign DEAF in the KK corpus, equivalent expressions are entirely absent, and DEAF in KK is used to refer to the entity 'deaf person' only. The fact that GEHÖRLOs ('deaf') has two meanings belonging to two different concept classes makes it difficult to categorise. When the sign is used in context, the syntactic slot serves to disambiguate between the two interpretations (see similar examples (29a) and (29b) in Section 2.4.2). However, considered in isolation, the meaning of GEHÖRLOS could either be vague/polysemous, or there could be two homophonous signs, meaning 'deaf' (as a property) and 'deaf person' respectively.

This theoretical debate is familiar from the PoS literature on spoken languages (e.g. Croft 2001) and cannot be resolved within the limited scope of this article. However, for a comprehensive PoS analysis, it will be helpful to distinguish between signs with several meanings from different concept classes, such as GEHÖRLOS, and signs with several related meanings from one and the same concept class. The latter situation commonly occurs in KK. The degree of lexical specificity often differs in DGS and KK, particularly with respect to event concepts. Where KK often has a single sign with a wide range of meaning depending on the context of the utterance, DGS employs several lexically differentiated items, such as in this example:

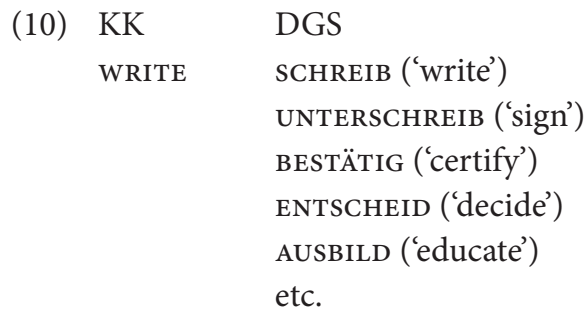




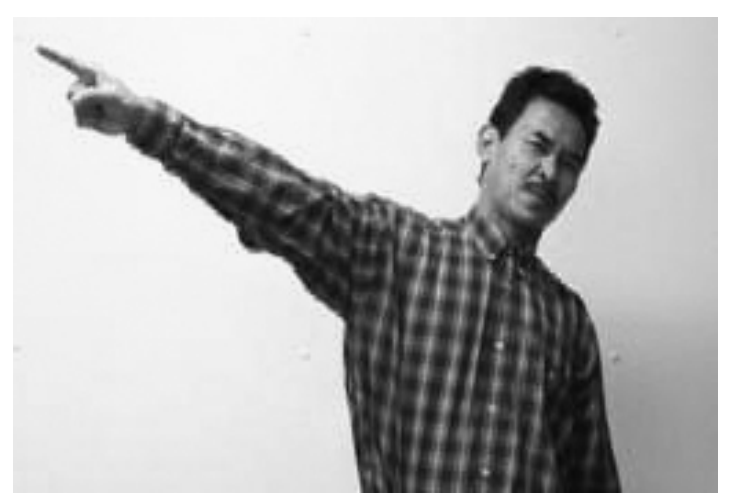

Figure 9. A pointing sign in Kata Kolok.

When the KK sign WRITE is used with reference to children, it can have the meaning 'learn, study in school', but in relation to an adult, it would mean 'teach'. However, all meanings of WRITE are semantically related and belong to the same main concept class, so there is no motivation for considering an analysis in terms of more than one lexical item.

Another interesting difference in the lexical organisation of DGS and KK is the almost total absence of place names in the latter. Place names are characterised by the semantic features [+proper, +concrete, 0 homogeneous, +individuated, -count, -animate, +local], and there are many such signs in DGS denoting countries (e.g. DeUtschland 'Germany'), cities (e.g. FRANKFURT), etc. However, KK has only a handful of place names at most. Usually, places are referred to by using a system of absolute spatial reference, which involves pointing to the exact direction of the target location (Marsaja 2008). This includes pointing behind oneself and pointing with fully outstretched arms (see Figure 9), both of which are unusual forms in DGS and other urban sign languages. It is uncommon in the local situation to talk about unfamiliar places, for instance in Europe, and obviously, it would be difficult to expand the system of absolute spatial reference beyond known locations. However, there are strategies around this problem if the need arises and which have not been fully investigated. For instance, signers may point to the direction of the nearest airport in order to refer to an unfamiliar place overseas, or to foreigners from overseas, signing, for instance, WHITEFOREIGNER INDEX ${ }_{\text {airport }}$ to refer to a European researcher visiting the village.

Clearly, the lexical and grammatical organisation of KK interface here in ways which are absent from DGS. The principle of absolute spatial reference through pointing makes lexical items in the category of place names unnecessary in KK. The grammar of pointing in KK is very complex, since there needs to be a way of specifying distance as well as direction, but the system has not been described in detail yet. ${ }^{10}$ In DGS, there is no such grammaticalised system of pointing, and therefore, the function of referring to place names must be handled by the lexicon. 
When working comparatively across several sign languages, both the language-independent semantic metalanguage and language-specific concept classification come into play. ${ }^{11} \mathrm{~A}$ more comprehensive investigation over larger data sets, testing systematically for a large number of semantic and structural features, can then yield principled results in the word class typology of sign languages. The next sections deal with criteria and classifications on the structural levels of syntax and morphology.

\subsection{Syntactic criteria for PoS differentiation}

\subsubsection{Criteria and classification on the syntactic level}

As pointed out by Anward (2001), there seems to be a basic characteristic mapping of semantic classes onto syntactic functions across languages (cf. similarly Croft 2001, which is based on substantial cross-linguistic evidence). Given the three major concept classes posited in the previous section, prototypical combinations of semantic class and syntactic function may be lexicalized in terms of 'traditional' parts of speech. The combination of entity class and argument function is lexicalized as nouns (11a), event class and predicate function as verbs (11b). The combinations of property class and modifier function are lexicalized by adjectives in argument modifier (attributive) function (11c) and by adverbs in predicate modifier (adverbial) function (11d) (cf. Anward 2001).
a. [entity; argument]
Noun
b. [event; predicate]
Verb
c. [property; argument modifier]
Adjective
d. [property; predicate modifier]
Adverb

Hengeveld (1992) argues that there is a lot of variation in part-of-speech differentiation across (spoken) languages in that, on the one hand, a language does not obligatorily use all syntactic functions, lacking, for instance, one or both modifier functions. On the other hand, a language may lexicalise any combination of semantic class and syntactic function other than the prototypical ones listed in (11) above. Consequently, concept classes may also be multiply lexicalized in non-prototypical syntactic functions (cf. Anward 2001).

In order to investigate which concepts of concept classes can be lexicalized in which syntactic functions, the part-of-speech definitions in Hengeveld (1992), given in a slightly modified form in (12), are useful as an initial point of reference.

(12) a. predicate use (p): items can, without special marking, be used as a predicate,

b. argument use (a): items can, without special marking, be used as an argument,

c. argument modifier use (am): items can, without special marking, be used as an argument modifier, 
d. predicate modifier use ( $\mathrm{pm})$ : items can, without special marking, be used as a modifier of a predicate or of another modifier.

Whether [entity; argument] and [event; predicate] need to be separately lexicalized in DGS and KK is first and foremost an empirical question. For DGS, Erlenkamp (2000) argues that the noun-verb distinction is neutralised because of the absence of a noun category. In her approach, the DGS lexicon is divided into three categories instead of the traditional parts of speech. There is a small class of 'directional verbs' comprising 13 signs, and another small group of signs in the class of 'classificatory verbs'. As in IPSL (cf. Section 1.3), directional verbs in DGS move between two grammatically significant spatial locations; this phenomenon is explained in more detail in Section 2.5. Classificatory verbs have a classifier handshape and include signs such as those in the examples in Figures 3 and 4 in Section 1.2. All other DGS signs belong to a large group of 'multifunctional signs' in Erlenkamp's analysis. The resulting correlation between lexical and syntactic categories in DGS can be represented in a simplified way as in Figure 10:

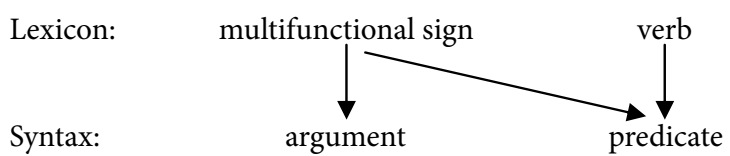

Figure 10. Correlation between lexical and syntactic categories in DGS according to Erlenkamp (2000).

However, this approach has been criticised in Keller, Pfau and Steinbach (2002) for its idiosyncratic selection of criteria defining the word classes. These include: 1. reference, 2. morphological distribution (of plural or local marking), 3. reduplication (e.g. intensity or duration of action), 4. valency, and 5. occurrence of lexical items in certain syntactic slots, such as in argument or predicate function. It is therefore difficult to use this approach as a basis of comparison for typological studies on word classes in other sign languages.

\subsubsection{Application of syntactic criteria to DGS and $K K$}

For the purpose of this article and until more detailed data become available, we discuss selected representatives from each one of the three main concept classes in this section and briefly point out tendencies for the two target sign languages which emerge from the preliminary findings.

On the basis of these criteria, signs can be mapped onto their syntactic functions as in Table 2. For this first pilot investigation, a small number of signs were chosen on the basis of a) being reasonably frequent in the data corpora, so that substantiated generalisations can be made, and b) having reasonably close semantic matches in both sign languages. Table 2 summarises a few illustrative examples. Some items 
in DGS (QUESTION in the entity class, and DEAF and HEARING in the property class) do not have equivalents in KK and the corresponding slots are therefore empty in the table. A question mark, which also occurs with a few KK items, means that despite the sign's relatively frequent occurrence, no conclusive evidence could be found yet in the data as to its semantics and/or structural status in utterances. For instance, it is not clear whether the sign FRIEND in KK, made with two intertwined index fingers in a to-and-fro movement, designates an entity or an event, and how the sign maps onto syntactic functions in the sentence. An utterance such as (13) could have several different interpretations. Interpretations (14a) and (14c) would assign FRIEND to an argument function, whereas in interpretation (14b), FRIEND would be in a predicate slot (cf. similar sentences in examples (22) to (24) below). Further tests are needed to conclusively decide the status of the KK sign FRIEND, and a number of other signs which are marked by question marks in Table 2 .

KK

(13) INDEX 1 FRIEND GOOD

(14) a. 'My friend is good/nice.'

b. 'I am befriending (someone), that is good.'

c. 'I have a friend.'

The DGS signs DEAF and HEARING occur twice in the table because their semantic content maps onto two different major concept classes, as already discussed. The DGS sign QUESTION is similar and formationally related to ASK, but the latter has obligatory verb agreement. In KK, there is a more general term SIGN-TALK (see Figure 11), which means 'communicate (in sign language)' and includes all kinds of communicative acts, such as telling, discussing, asking, and so forth.

As the table shows, there are striking differences with respect to the occurrence of concepts and concept classes in syntactic slots in both sign languages. Members of the

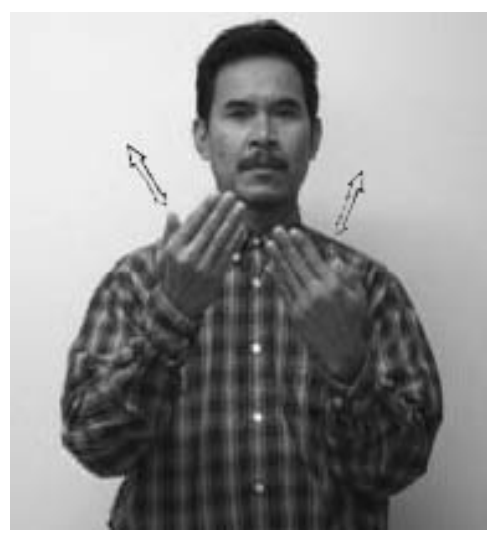

Figure 11. SIGN-TALK in Kata Kolok. 
Table 2. Occurrences of entity, event, and property concepts in syntactic functions.

\begin{tabular}{|c|c|c|c|c|c|c|c|c|c|}
\hline \multirow{2}{*}{$\begin{array}{l}\text { semantic } \\
\text { classes }\end{array}$} & \multirow{2}{*}{ sign examples } & \multicolumn{4}{|c|}{ DGS } & \multicolumn{4}{|c|}{ Kata Kolok } \\
\hline & & $\mathrm{p}$ & $\mathrm{a}$ & $\mathrm{am}$ & $\mathrm{pm}$ & $\mathrm{p}$ & $\mathrm{a}$ & $\mathrm{am}$ & $\mathrm{pm}$ \\
\hline \multirow{6}{*}{ entity } & DEAF / HEARING & + & + & & & + & + & & \\
\hline & FEMALE & + & + & & & & + & & \\
\hline & CAR & & + & & & & + & & \\
\hline & FRIEND & + & + & & & & $?$ & & \\
\hline & QUESTION & & + & & & & & & \\
\hline & FOOD & & + & & & & $?$ & & \\
\hline \multirow{7}{*}{ event } & SIGN-TALK & + & & & & + & & & \\
\hline & ASK/REQUEST & + & & & & + & & & \\
\hline & STAY & + & & & & + & & & \\
\hline & DIE & + & & & & + & & & \\
\hline & CAR-DRIVE & + & & & & + & & & \\
\hline & EAT & + & & & & + & & & \\
\hline & WORK & + & + & & & + & & & \\
\hline \multirow{4}{*}{ property } & DEAF / HEARING & & & + & & & & & \\
\hline & SMALL & + & & + & & + & & $?$ & \\
\hline & BAD / GOOD & + & & + & + & + & & & \\
\hline & BLACK & + & & + & & + & & & \\
\hline
\end{tabular}

entity concept class in DGS can also be used predicatively, but this is almost never the case in KK. Instead, KK seems to be closer to the prototypical mapping for nouns as the combination [entity; argument], as in (12a). Further characteristics of a putative "noun" category in KK would need to be checked against the entity class as a whole.

Interestingly, the DGS lexicon includes an abstract entity concept FRAGE ('question') (15a), which occurs in argument function without the obligatory verb agreement morphology of its verbal counterpart FRAG ('ask') (15b). ${ }^{12}$ Whereas DGS has lexicalised both [event; predicate] and [entity; argument] for this concept (see Figures 12-14), the same concept in KK only occurs as [event; predicate], and there is no entity-like abstraction.

\section{DGS}

(15) a. POSS ${ }_{2}$ QUESTION INDEX $_{1}$ REALLY UNDERSTAND ${ }^{\wedge}$ NEG

'I really do not understand your question.'

b. POSS $_{1}$ FRIEND $_{3}$ ASK $_{1}$

'My friend asks me.'

In contrast to Indo-European languages, both sign languages primarily lexicalise the concept 'deaf' as a prototypical noun [entity; argument], which can also be used predicatively. This illustrates that any lexicalisation is culturally determined as well. DGS has further lexicalised a property concept 'deaf' in attributive use, probably through language contact with German. The following examples illustrate the use of DEAF in DGS and KK. (16b) shows the ungrammaticality of using DEAF as a modifier in KK. 


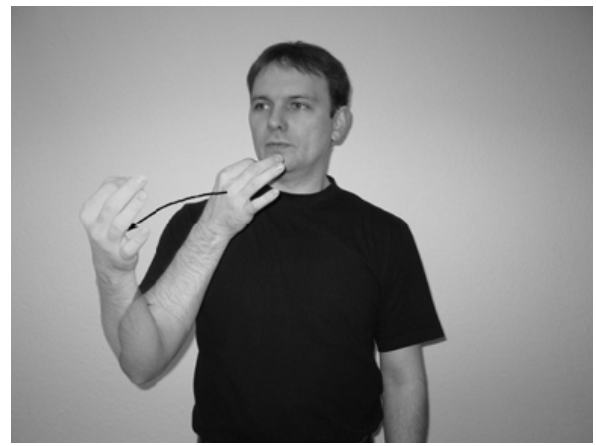

Figure 12. $\mathrm{DGS}_{1} \mathrm{FRAG}_{3}$ ('I ask someone').

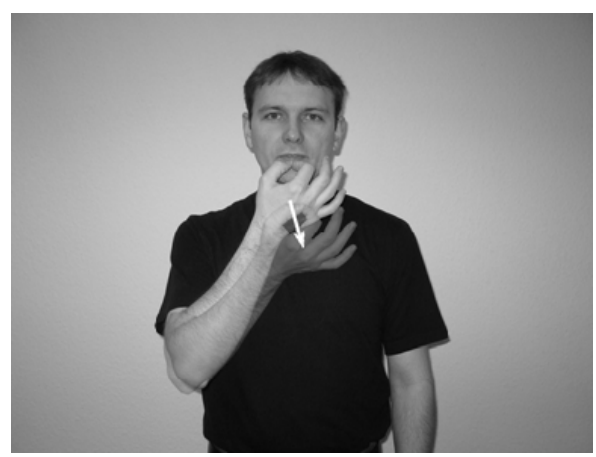

Figure 14. DGS FRAGE ('question').

KK
a. DEAF COME
'A deaf person came.'
b. *FEMALE DEAF COME
'A deaf woman came.'

\section{DGS}

(17) DEAF FRIEND ${ }_{x}$ PERSON-CL ${ }_{y}$ :'one comes here' 'A deaf friend comes to me.'

The syntactic use of property concepts in KK is also much more restrictive than that in DGS. Property concepts in KK seem to be used as predicates only, as illustrated in the following examples:

\section{KK}

(18) a. CHICKEN INDEX ${ }_{x}$ SMALL, DEAD

'There was a chicken there, which was small. It was dead.'

b. * SMALL CHICKEN DEAD

'A small chicken is dead.' 


\section{DGS}

(19) BIRD SMALL DEAD

'A small bird is dead'.

In addition to their use in predicate function, the DGS property signs GUT ('good') and SCHLECHT ('bad') can be used as both argument and predicate modifiers (examples (20) and (21)), whereas the semantic equivalents in KK are used in predicate function only, often following a whole phrase/sentence functioning as the argument (22).

DGS

(20) POSS 1 WIFE WORK GOOD

'My wife works well.'

DGS

(21) INDEX $_{1}$ BEEN GOOD RESTAURANT ${ }_{1}$ VISIT $_{\mathrm{x}}$

'I have been to a good restaurant.'

KK

(22) INDEX 1 DEAF GATHER, GOOD

'It is nice when I get together with deaf people.'

The KK sign GOOD is also used in a possessive construction in the predicate slot and then has a possessive meaning. In possessive constructions in KK, the sign GooD has become grammaticalised and does not necessarily have a literal meaning. While example (23) is ambiguous between the lexical and the grammatical function of GooD, the context in an utterance such as (24) clearly indicates that GOOD has lost its lexical meaning and is functioning in a possessive reading only.

KK

(23) INDEX $_{1}$ FEMALE MARRY GOOD

'I have a wife, (which is good).'

(24) INDEX 1 FEMALE MARRY GOOD, CRAZY

'I have a wife. She is crazy'.

However, KK does not completely lack signs that can be used as modifiers. For instance, in (25) MUCH is used as predicate modifier. Moreover, the morphology of KK includes processes of marking the comparative in entity concepts (see Section 2.5).

KK

(25) INDEX 1 GO-AWAY WORK MUCH COME GIVE MONEY, GOOD\#INTS

'I go and work a lot to bring money (to my family), and that is great.'

In DGS, the sign ARBEIT ('work') can occur in argument function in addition to the prototypical predicate slot: 


\section{DGS}

(26) WORK FIND DIFFICULT\#INTS

'It is very difficult to find a job.'

In such a context, it is worthwhile asking whether ARBEIT retains its original event semantics or has become an abstract entity. It seems that ARBEIT occurs in the combinations [event; predicate] and [event; argument] only, without any special marking differentiating between the two usages. Similar cases can also be found in English, as in the examples in (27), where (27b) acts as a bridge context. The re-arrangement of categories here proceeds in two stages, where [event; argument] is intermediate between the two prototypical assignments.

(27) a. It is so stressful to work nowadays. ( $\quad$ work $_{\mathrm{V}}$ [event; predicate])

b. Work is so stressful nowadays. $\quad\left(\right.$ work $_{\mathrm{N}}$ [event; argument])

c. I showed my work to my boss, but he didn't like it.

$\left(\right.$ work $_{\mathrm{N}}$ [entity; argument])

In DGS, ARBEIT ('work') does not seem to occur in contexts where it has clearly lost its event semantics and is conceptualised as an entity. Further evidence for the event character of the sign ARBEIT when used in an argument slot comes from the fact that, unlike other entity concepts, it is not compatible with possessive pronouns (examples $28 \mathrm{a}-\mathrm{b})$. Instead, a personal pronoun is used (28c).

\section{DGS}

(28) a. POSS $_{2}$ CAR GOOD

'Your car is good'.

b. ${ }^{*}$ POSS $_{2}$ WORK GOOD

c. INDEX 2 WORK GOOD

'Your work is good./You work well.'

Whereas ARBEIT ('work') in DGS always has an event meaning no matter in which semantic slot it occurs, the sign GEHÖRLOs ('deaf') can have either an entity meaning ('deaf person') or a property meaning, the latter particularly when used in a modifier slot, as in (29a). In predicative use, GEHÖRLOs has an entity meaning only (29b).

\section{DGS}

$$
\begin{aligned}
& \text { a. DEAF FRIEND } \\
& \text { 'a deaf friend' } \\
& \text { *'a deaf person, a friend' } \\
& \text { b. INDEX } x_{\mathrm{x}} \text { DEAF } \\
& \text { 'S/he is a deaf person.' }
\end{aligned}
$$

As mentioned in the discussion in 2.3.3, it is difficult to decide here on theoretical grounds whether the sign GEHÖRLOs ('deaf') is polysemous, or whether there are two homophonous signs, each with its own meaning. 
In the limited context of this article, we do not go into details of which lexical categories are lexicalised and mapped onto which syntactic functions in DGS and KK. This would necessitate an extensive analysis on the basis of a large data corpus from both languages, which is yet to be undertaken.

\subsection{Criteria and classification on the morphological level}

Languages obviously differ greatly from one another at the morphological level, and therefore, morphological criteria for word class assignment must be determined language-specifically for each language. The degree to which sign languages can differ from one another at the morphological (and also the syntactic) level has not been properly recognised even in the sign language linguistics literature, mainly because there is very little information about non-Western, and also non-urban, sign languages. The better-documented urban sign languages in industrialised countries have been found to have many morphological processes in common (e.g. expression of agreement, aspectual marking, cf. Aronoff et al. 2005). In particular, the morphology of these sign languages is largely simultaneous rather than sequential, and involves modulations in signing space typical of the sign language modality. However, comparing a wider range of typologically dissimilar sign languages does reveal a larger degree of cross-linguistic differentiation (cf. Zeshan 2005, 2006).

In this section, we consider morphological properties of DGS, RSL and KK, also drawing on Schwager (2004) for the discussion of the morphology of RSL as well as the morphological typology of sign languages in general. With respect to the morphological level within the topic of parts of speech, the approach used here is different from earlier approaches such as Zeshan (2000) and Erlenkamp (2000) in that we first establish an extensive inventory of morphological processes, exploring a full range of theoretically possible and cross-linguistically instantiated types of morphology. This inventory is then tested against sign language data from typologically diverse sign languages, observing the morphological behaviour of a substantial number of semantically equivalent signs.

Sign languages have complex morphologies, but many of their morphological processes are uncommon, unattested or even logically impossible in spoken languages. Since it would go far beyond the scope of this article to explain the various kinds of sign language morphology in detail, we can only attempt a very general overview and give a few illustrative examples in order to demonstrate how morphological criteria bear on parts of speech categorisations in sign languages.

A particularly striking example of different categorisation for DGS/RSL and KK at the morphological level concerns the category of directional verbs, also known as agreement verbs. These verbs, already mentioned in Section 2.4.1, have been discussed extensively in the sign linguistics literature (e.g. Meir 1998; Mathur 2000; Padden 1990). Directional verbs in DGS and RSL inflect for subject and object in one of 


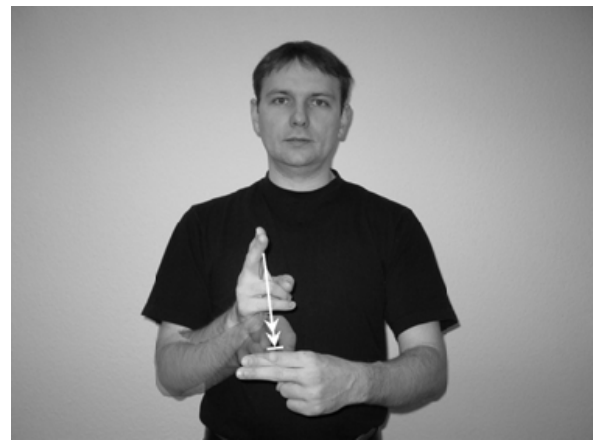

Figure 15. RSL ${ }_{1}$ DISTURB $_{2}$ ('I disturb you').

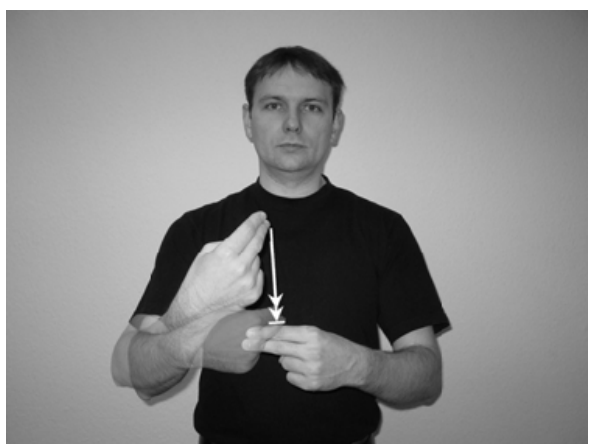

Figure 16. RSL ${ }_{2}$ DISTURB $_{1}$ ('you disturb me').

two ways: they either take subject agreement prefixes and indirect object agreement suffixes which consist of spatial locations at the beginning and the end point of the predicate sign's movement respectively (see Figures 12 and 13 in Section 2.4.2), or they inflect internally through featural alteration, changing only the orientation of the hand in terms of which way the palm and/or fingertips are facing (see Figures 15 and 16).

Thus the number and role of arguments that a verb may take, that is, the verb's valency, is overtly expressed through subject/object affixation in DGS. If an [event; predicate] in DGS is used transitively, the spatial subject/object affixes must be added if this option is available for the particular sign. However, many semantically transitive signs in DGS do not participate in this kind of morphology and do not allow the addition of agreement affixes. Therefore, if a transitive [event; predicate] cannot apply the spatial agreement morphology, it requires an auxiliary verb called PAM (person agreement marker, Rathmann (2000), see Figure 17). The PAM auxiliary then takes on valency-induced agreement morphology, as in example (30) below.

\section{DGS}

MOST DEAF HATE\#INTS PAM 3 TEACHER

'Most deaf people hate teachers (at deaf schools) very much.'

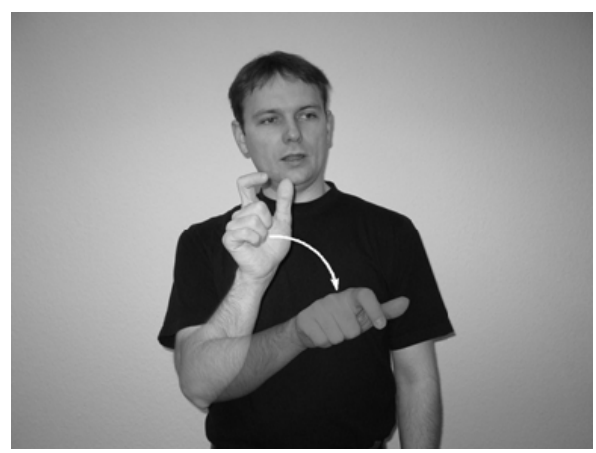

Figure 17. ${ }_{1} \mathrm{PAM}_{3}$ in German Sign Language. 
While valency is lexically inherent in both DGS and KK signs, it can be morphologically marked in DGS only. In KK, there is no class of directional/agreement verbs and no auxiliary, so that the transitivity of [event; predicate] cannot be expressed overtly at the morphological level. Events in predicate use may be used as transitive or intransitive without any morphological changes in KK. ${ }^{13}$ For instance, ASK/REQUEST [event; predicate] in KK is transitive and requires a subject and an object, but the sign's transitivity/valency does not have consequences at the morphological level. Figure 18 shows the transitive predicate sign ASK/REQUEST used in example (31). The form of the sign does not vary according to who is the subject or the object of the sentence, since no process of adding a directional movement to the sign exists to express transitivity in KK. Unlike ASK/REQUEST in KK, the semantically equivalent sign in DGS does have a directional movement, as indicated by the subscript in the transcription of example (32). ${ }^{14}$

\section{KK}

(31) PAST-TIME INDEX 1 MONEY ASK/REQUEST NEG

'I did not ask (someone) for money.'

DGS

(32) INDEX 1 MONEY BEG\#DISTR ${ }_{3}$ NEVER

'I never went around begging for money'.

Without going into details of sign language morphology here, Table 3 lists a substantial number of morphological processes, comparing data from DGS, RSL and KK. Similarly to the link between semantic classes and syntactic slots in Section 2.4.2, Table 3 links semantic classes and morphological processes. The classification of morphological processes in the third column of the table assumes that there is a typology of phonological representation of morphology in sign languages, whereby sign language morphology is subdivided into intrasegmental, suprasegmental, and segmental morphology, the

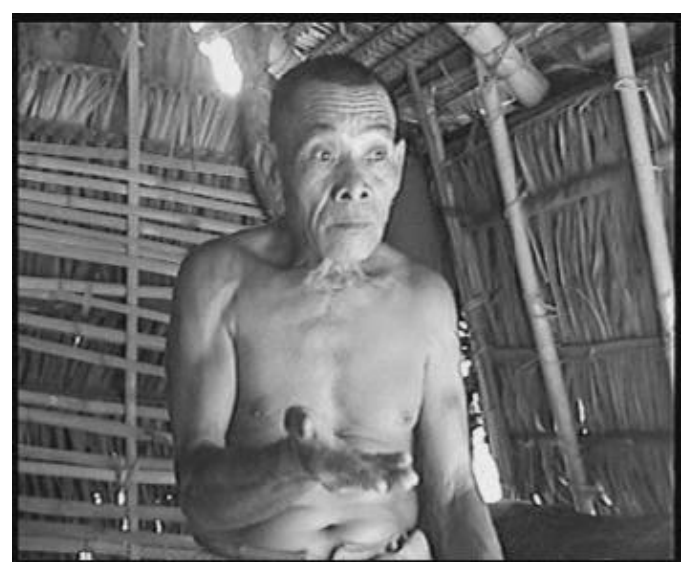

Figure 18. ASK/REQUEST in Kata Kolok. 
Table 3. Outline of morphological criteria in DGS, RSL, and KK.

\begin{tabular}{|c|c|c|c|c|c|}
\hline concept classes & $\begin{array}{l}\text { grammatical } \\
\text { categories }\end{array}$ & $\begin{array}{l}\text { morphological } \\
\text { processes }\end{array}$ & DGS & RSL & KK \\
\hline \multirow{13}{*}{ event } & subj./obj. & (1) affixation & + & + & - \\
\hline & agreement & (2) featural alteration & + & + & - \\
\hline & distributive & (3) reduplication & + & + & - \\
\hline & reciprocal & (4) reduplication & + & + & - \\
\hline & \multirow{2}{*}{ negation } & (5) affixation & + & + & - \\
\hline & & (6) suprafixation & + & - & - \\
\hline & \multirow{3}{*}{ aspect } & (7) reduplication & + & + & - \\
\hline & & (8) affixation & - & + & - \\
\hline & & (9) featural alteration & - & - & - \\
\hline & intensive & (10) featural alteration & + & + & + \\
\hline & diminutive & (11) featural alteration & $?$ & + & $?$ \\
\hline & $\operatorname{mood}$ & (12) suprafixation & + & + & + \\
\hline & class agreement & (13) affixation & + & + & + \\
\hline \multirow{2}{*}{ entity } & number & (14) reduplication & + & + & $?$ \\
\hline & locus (agreement) & (15) affixation & + & + & - \\
\hline \multirow{2}{*}{ property } & \multirow{2}{*}{ comparative } & (16) featural alteration & + & + & + \\
\hline & & (17) suprafixation & + & + & + \\
\hline
\end{tabular}

latter again subdivided into sequential (or concatenative) and simultaneous morphology (cf. Schwager 2004). Sign languages have both form-building morphology for grammatical categories such as subject/object agreement and aspect/aktionsart marking, and sign-building morphology which creates new signs, for instance by way of compounding. ${ }^{15}$ Only the former is represented in Table 3.

The types of morphological processes represented in Table 3 include affixation ( 1 , $5,8,13$ and 15 in the third column), featural alteration $(2,9,10,11$ and 16), reduplication $(3,4,7$ and 14) and suprafixation $(6,12,17)$. They relate to the three levels of phonological representation of sign language morphology mentioned above in the following way:

(33) a. Featural alteration is an intrasegmental morphological process.

b. Suprafixation is a suprasegmental morphological process.

c. Reduplication and affixation are segmental morphological processes; they can be simultaneous or sequential.

An example of featural alteration has already been discussed in connection with the category of agreement verbs (cf. no. 2 in Table 3). Since the orientation of palm and fingers can be considered a feature of the handshape in such a sign, changing this orientation is equivalent to an intrasegmental feature alteration, much like the umlaut found in some Germanic languages. For class agreement (no. 13 in the table), the 
handshape representing the referent class, such as the human and vehicle referents in Figures 3 and 4, is a bound morpheme that is combined with morphemes of location and movement in an affixation process, whereby a complex multimorphemic sign is created. Suprafixation occurs when manual signs are combined with non-manual signals, which can spread over more than one sign. For instance, in many sign languages a side-to-side headshake signalling negation combines with the predicate sign and can then spread over other signs, maximally co-occurring with the entire utterance (Zeshan 2004). As has been argued by Pfau (2002), the headshake can be considered affixal to the verb in such cases, but behaves like a suprasegmental, "intonational" feature at the formational, phonological level (cf. no. 6 in the table).

In a few cases, it is not clear from the data yet whether a morphological process occurs systematically. For instance, this is the case for diminutive marking of event concepts in DGS and KK (no. 11 in the table). The diminutive is the counterpart of the intensive marking and denotes a weak instantiation of an event. For instance, the sign CRY in RSL can receive an intensive marking to mean something like 'cry one's eyes out, weep bitterly', or a diminutive marking meaning 'cry a little, shed a few tears'. In both cases, there is a slight internal difference in the movement component of the sign (featural alteration in the movement phoneme), and there is an additional nonmanual marking (suprafixation) which has not been included in the simplified Table 3. The diminutive occurs with a number of event lexemes in RSL (though by no means with all of them), but it is not clear whether an equivalent process exists systematically in DGS and KK.

In spoken languages, affixation and reduplication are sequential processes adding morphemes either before or after a stem. While this occurs in sign languages as well, it is more common for segmental morphology in sign languages to be simultaneous. For instance, a one-handed sign can be reduplicated by mirroring its movement on the second hand at the same time, which is used to express dual and reciprocal categories in many sign languages (cf. no. 4 in the table and Figure 19). When the spatial location of a sign is changed for grammatical purposes, such as signing HOUSE not in the neutral location in front of the signer, but on the far right-hand side, for instance, the

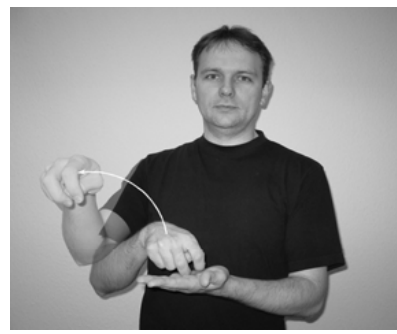

a.

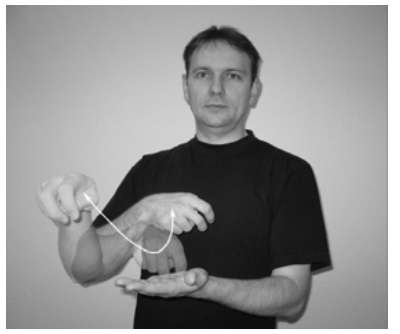

b.

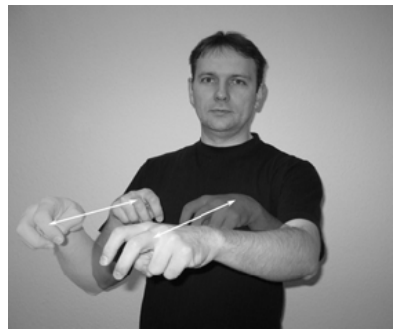

c.

Figure 19. RSL sign ${ }_{1}$ SEND-LETTER $_{3}$ (19a), reduplicated sequentially (19b) and simultaneously (19c). 


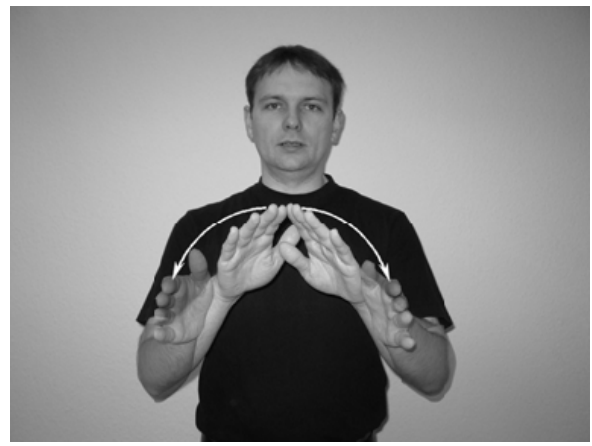

a.

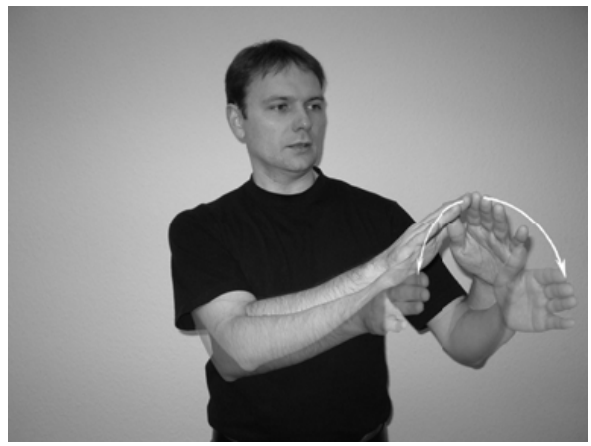

b.

Figure 20. DGS sign HOUSE in neutral location (20a) and with locus affix (20b).

grammatically significant spatial location on the right side can be regarded as an affix signaling locus agreement, which is produced simultaneously with the sign HOUSE itself (cf. no. 15 in the table and Figure 20).

Comparing the kinds of morphological processes that are found in DGS, RSL and KK, a number of general tendencies appear at first sight. The morphologies of DGS and RSL are much more similar to one another than to KK, with only a few different values for DGS and RSL. This is in line with the previous findings of sign language linguistics which argue for a lot of morphological similarities across sign languages, chiefly on the basis of data from North American and European sign languages. Secondly, by far the largest number of morphological processes relates to events, but KK expresses far fewer grammatical categories at the morphological level than DGS and RSL. Morphology relating to the entity concept class is also largely absent in KK, with only one questionable category (no. 14 in the table). Finally, it is of interest that property concepts have associated morphology in all three sign languages and thus behave identically in this domain, although the actual set of signs falling into the property class is not the same across the target sign languages (cf. Section 2.3.3). KK signs for property concepts participate in the same kinds of morphological processes for marking the grammatical category of comparative as DGS signs for property concepts (no. 16 and 17 in the table), but unlike in DGS, the KK signs tend not to appear in the syntactic positions of either argument or predicate modifier (cf. Section 2.4.2).

At this stage of research, it is too early to demonstrate conclusively how the morphological differences between DGS, RSL and KK relate to their respective PoS systems. A much more extensive survey based on a much larger data set will be necessary to consolidate the initial results. In general, the above findings suggest that morphological operations may play a greater role in distinguishing between different word classes in sign languages like DGS and RSL, which have a larger array of morphological processes to draw on. This is exemplified, for instance, by the class of agreement verbs in DGS and RSL. Moreover, the fact that KK is a more "isolating" language than DGS and RSL is a real typological difference and has a direct bearing on the search for 
word class categories in $\mathrm{KK}$, where morphological criteria will play a much lesser role in word class assignment. Whatever further investigation may reveal, the present study certainly demonstrates the importance and the methodological viability of looking at a comprehensive range of morphological processes rather than arbitrarily choosing only a limited set of morphological criteria.

\section{Conclusion}

This article has explored a unified approach studying PoS systems across typologically diverse sign languages. Both language-independent semantic features and languagespecific morphosyntactic criteria play an important role in this undertaking. Rather than having an ad hoc mix of criteria at various levels, a shortcoming that previous attempts have suffered from, the present approach allows for a principled way of approaching the lexical categorisations inherent in diverse sign languages. At the same time, the data upon which the emerging generalisations are based are taken directly from large transcribed corpora of spontaneously produced sign language data, thus keeping the theoretical work close to the primary data. Since there is so little established tradition and consolidated knowledge related to parts of speech systems in sign languages, this aspect of the approach is particularly important.

Further research that will build upon the preliminary results presented in this article is already inherent in the methodological and theoretical issues addressed in Section 1 of this article. First of all, the complete 250-word data sets should be compiled for all three target sign languages and checked against the large number of features that have been detailed above. Secondly, "difficult" sign types, such as the notoriously complicated classifier signs mentioned in Section 1.2, also need to be included in the analysis, as well as closed lexical classes in addition to the open classes discussed here. If the analysis tools prove fruitful for the initial three sign languages, the same methodology could then be used to investigate aspects of PoS systems in other sign languages. Over time, it should be possible to achieve coherent PoS characterisations compiling information from all relevant levels, semantic, syntactic and morphological. Comparing these to PoS systems in spoken languages will then be of great potential interest for the study of linguistic typology and language universals.
Abbreviations
${ }_{x}$ PERSON-CL ${ }_{\mathrm{y}}: \ldots, \quad$ human classifier, with locus marking and meaning between quotes $\operatorname{INDEX}_{1} \quad$ personal pronominal marker in first person
$\operatorname{INDEX}_{\mathrm{x}} \quad$ indexical marker, with locus marking
INTS intensifier
DIST distributive 


\begin{tabular}{|c|c|}
\hline NEG & negative \\
\hline $\mathrm{PAM}_{3}$ & person agreement marker, with locus marking in third person \\
\hline POss $_{1}$ & possessive pronominal, with first person marking \\
\hline POSS $_{2}$ & possessive pronominal, with second person marking \\
\hline${ }_{3}$ GLOSS $_{1}$ & $\begin{array}{l}\text { directional sign with subject agreement prefix in third person and object } \\
\text { agreement suffix in first person }\end{array}$ \\
\hline GLOSS-GLOSS & multi-word gloss representing a single sign \\
\hline $\mathrm{GLOSS}^{\wedge} \mathrm{GLOSS}$ & sequential morphemes \\
\hline ILOSS\#GLOSS & simultaneous morphemes \\
\hline
\end{tabular}

\section{Acknowledgements}

We are indebted to Roland Pfau and to an anonymous reviewer for useful comments on the first draft of this article. We would also like to express our deep gratitude to the Kata Kolok using village community in Bali for allowing us to do research in their village and to make use of their video data in publications. This study is part of a larger research project on "Sign Language Typology - The Cross-Linguistic Study of Sign Languages", which is supported by the German Research Council (Deutsche Forschungsgemeinschaft, DFG), grant no. ZE507/1-3.

\section{Notes}

1. It is a common convention in much of the literature on German Sign Language to use glosses in German. Where no such convention exists, as is the case for Kata Kolok as well as some other sign languages discussed in this article, English glosses are generally used. For convenience, however, we also use English glosses for examples of German Sign Language sentences in Sections 2.4 and 2.5 .

2. Spoken languages also have words that "sound like" what they mean, but these sound-symbolic words, such as roar, rumble, swish, beep, etc. in English, are marginal in the lexicon. In sign languages, a major part of the lexicon consists of such words, and they cannot therefore be ignored as marginal cases.

3. The term "classifier" is widely used in sign language linguistics, although the phenomena it covers are different from the use of the same term in spoken language linguistics.

4. Statistically and without the genetic factor, a community of this size would be expected to have only one or two deaf people.

5. For the purpose of this article, we are not considering additional possible factors at the phonological and the discourse levels. For example, it has been suggested that in DGS, the silent mouth movements derived from spoken German words play a role in distinguishing between nouns and verbs (see Boyes-Braem and Sutton-Spence (2001) for details about mouth movements in various sign languages). Discourse pragmatic features could be relevant, for instance, in connection with indexical signs, where there is a difference between the physical presence or absence of referents in the signing environment. 
6. "A concept class is a bundle of interrelated concepts that can be defined by a superordinate feature (usually a hyperonym)."

7. We use the term 'entity' as a concept class instead, with a semantic range including person/ animal/plant/thing as well as abstract entities.

8. Wilbur (in press) even goes further by arguing that the formational aspects of ASL signs that reflect semantic telicity should actually be regarded as morphemes.

9. Note that for want of better terms, some terms occur at two different levels, once in a wider sense (i.w.s.) and once in a narrower sense (i.n.s.).

10. Decoding the complex system of pointing in $\mathrm{KK}$ is the central theme of a current research project on KK at the Max Planck Institute for Psycholinguistics.

11. This applies to closed classes of 'function words' as well as open classes. For instance, not all sign languages differentiate between a basic clause negator 'not' and more specific negators with aspectual meanings, such as 'not yet', 'not any more' (Zeshan 2004).

12. Verb agreement morphology is explained in more detail in Section 2.5.

13. KK does have one pair of formationally related signs generally denoting 'giving' or 'taking', which seem to have a directional movement. However, we cannot say with certainty at this stage whether these signs are morphologically complex or simply constitute two different lexical entries.

14. In this example, the verb agrees with the object (third person distributive) only.

15. Form-building morphology includes all processes of inflection, whereas sign-building morphology refers to processes that create new lexical items, such as derivation and compounding.

\section{References}

Anward, J. 2000. A dynamic model of part-of-speech differentiation. Approaches to the typology of word classes, Bernard Comrie \& Petra Vogel (eds.), 3-45. Berlin: Mouton de Gruyter.

Anward, J. 2001. Parts of speech. Language typology and language universals: An international handbook. Vol. 1, Martin Haspelmath; Ekkehard König; Wulf Oesterreicher \& Wolfgang Raible (eds.), 726-735. Berlin: Mouton de Gruyter.

Aronoff, Mark; Irit Meir \& Wendy Sandler. 2005. The paradox of sign language morphology. Language 81(2): 301-344.

Boyes Braem, Penny \& Rachel Sutton-Spence (eds.). 2001. The hands are the head of the mouth: The mouth as articulator in sign languages. Hamburg: Signum.

Branson, Jan; Don Miller; Gede Marsaja \& Wayan Negara. 1996. Everyone here speaks sign language, too: A deaf village in Bali, Indonesia. Multicultural aspects of sociolinguistics in deaf communities, Ceil Lucas (ed.), 39-57. Washington, DC: Gallaudet University Press.

Bybee, Joan. 2000. Verb. Morphology: An international handbook on inflection and word-formation. Vol. 1, Geert Booij; Christian Lehmann \& Joachim Mugdan (eds.), 794-808. Berlin: Mouton de Gruyter.

Comrie, Bernard. 1976. Aspect. Cambridge: Cambridge University Press. 
Croft, William. 2001. Radical construction grammar. Syntactic theory in typological perspective. Oxford: Oxford University Press.

Dixon, Robert M.W. 1982. Where have all the adjectives gone? Where Have All the Adjectives Gone? and other essays in Semantics and Syntax, Robert Dixon, 1-62. Berlin: Mouton Publishers.

Emmorey, Karen (ed.). 2003. Perspectives on classifier constructions in sign languages. Mahwah, NJ: Lawrence Erlbaum.

Erlenkamp, Sonja. 2000. Syntaktische Kategorien und lexikalische Klassen: Typologische Aspekte der Deutschen Gebärdensprache. München: Lincom.

Evans, Nicholas. 2000. Word class in the world's languages. Morphology: An international handbook on inflection and word-formation. Vol. 1, Geert Booij; Christian Lehmann \& Joachim Mugdan (eds.), 674-732. Berlin: Mouton de Gruyter.

Fernald, Theodore B. \& Donna J. Napoli. 2000. Exploitation of morphological possibilities in signed languages: Comparison of American Sign Language with English. Sign Language \& Linguistics 3(1): 3-58.

Hengeveld, Kees. 1992. Parts of speech. Layered structure and reference in a functional perspective, Michael Fortescue; Peter Harder \& Lars Kristoffersen (eds.), 29-56. Amsterdam: John Benjamins.

Heßmann, Jens. 2001. GEHÖRLOS SO!: Materialien zur Gebärdensprache. Hamburg: Signum.

Johnston, Trevor. 2001. Nouns and verbs in Auslan (Australian Sign Language): an open or shut case? Journal of Deaf Studies and Deaf Education 6(4): 235-257.

Keller, Jörg; Roland Pfau \& Markus Steinbach. 2002. Book review: Sonja Erlenkamp, Syntaktische Kategorien und lexikalische Klassen: Typologische Aspekte der Deutschen Gebärdensprache. Lincom Europa, 2000. Sign Language \& Linguistics 5(2): 247-253.

Lehmann, Christian \& Edith Moravcsik. 2000. Noun. Morphology: An international handbook on inflection and word-formation. Vol. 1, Geert Booij; Christian Lehmann \& Joachim Mugdan (eds.), 732-757. Berlin: Mouton de Gruyter.

Löbner, Sebastian. 2002. Understanding semantics. London: Arnold [Understanding Language Series]. (German edition: Sebastian Löbner. 2003. Semantik. Eine Einführung. Berlin: Mouton de Gruyter).

Marsaja, Gede. 2008. Kata Kolok - A deaf village and its sign language in Bali, Indonesia. Nijmegen: Ishara Press.

Mathur, Gaurav. 2000. Verb agreement as alignment in sign languages. PhD thesis, MIT (MIT Working Papers in Linguistics).

Meir, Irit. 1998. Syntactic-semantic interaction in Israeli Sign Language verbs: The case of backwards verbs. Sign Language \& Linguistics 1(1): 3-37.

Padden, Carol. 1988. Interaction of morphology and syntax in American Sign Language. New York, NY: Garland Publishing.

Padden, Carol. 1990. The relation between space and grammar in ASL verb morphology. Sign language research: theoretical issues, Ceil Lucas (ed.), 118-132. Washington, DC: Gallaudet University Press.

Pfau, Roland. 2002. Applying morphosyntactic and phonological readjustment rules in natural language negation. Modality and structure in signed and spoken languages, Richard P. Meier, Kearsy A. Cormier \& David G. Quinto-Pozos (eds.), 263-295. Cambridge: Cambridge University Press.

Rathmann, Christian. 2000. Does the presence of a person agreement marker predict word order in sign languages? Paper presented at the 7th International Conference on Theoretical Issues in Sign Language Research. July 2000, Amsterdam, Manuscript. 
Sandler, Wendy. 1999. Cliticization and prosodic words in a sign language. Studies on the phonological word, Tracy A. Hall \& Ursula Kleinhenz (eds.), 223-254. Amsterdam: John Benjamins.

Sandler, Wendy \& Diane Lillo-Martin. 2006. Sign language and linguistic universals. Cambridge: Cambridge University Press.

Sasse, Hans-Jürgen. 1993. Das Nomen - eine universale Kategorie? Sprachtypologie und Universalienforschung 46: 187-221.

Schembri, Adam. 2003. Rethinking 'classifiers' in signed languages. Perspectives on classifier constructions in sign languages, Karen Emmorey (ed.), 3-34. Mahwah, NJ: Lawrence Erlbaum.

Schwager, Waldemar. 2004. Polymorphemische Gebärden in der Russischen Gebärdensprache. MA thesis, University of Amsterdam.

Stassen, Leon. 1997. Intransitive predication. Oxford: Oxford University Press.

Supalla, Ted \& Elissa L. Newport. 1978. How many seats in a chair? The derivation of nouns and verbs in American Sign Language. Understanding language through sign language research, Patricia Siple (ed.), 91-132. New York, NY: Academic Press.

Van Valin, Robert. 2001. Syntax. Cambridge: Cambridge University Press.

Vendler, Zeno. 1967. Linguistics in philosophy. Ithaca, NY: Cornell University Press.

Wilbur, Ronnie B. In press. Complex predicates involving events, time and aspect: Is this why sign languages look so similar? Signs of the time. Selected papers from TISLR 2004, Josep Quer (ed.). Hamburg: Signum.

Zeshan, Ulrike. 2000. Gebärdensprachen des indischen Subkontinents. München: Lincom.

Zeshan, Ulrike. 2002. Towards a notion of 'word' in sign languages. Word: A cross-linguistic typology, Robert M.W. Dixon \& Alexandra Y. Aikhenvald (eds.), 153-179. Cambridge: Cambridge University Press.

Zeshan, Ulrike. 2004. Hand, Head and Face - Negative Constructions in Sign Languages. Linguistic Typology 8(1): 1-58.

Zeshan, Ulrike. 2005. Sign languages. The world atlas of language structures, Martin Haspelmath; Matthew S. Dryer; David Gil \& Bernard Comrie (eds.), 558-567. Oxford: Oxford University Press.

Zeshan, Ulrike (ed.). 2006. Interrogative and negative constructions in sign languages. Nijmegen: Ishara Press.

Zwitserlood, Inge. 2003. Classifiying hand configurations in Nederlandse Gebarentaal. Utrecht: LOT.

\section{Authors' address}

Ulrike Zeshan

International Centre for Sign Languages and Deaf Studies

Faculty of Arts, Humanities, and Social Sciences

Livesey House LH212

University of Central Lancashire

Preston PR1 2HE UK

United Kingdom

uzeshan@uclan.ac.uk, Waldemar.Schwager@t-online.de 